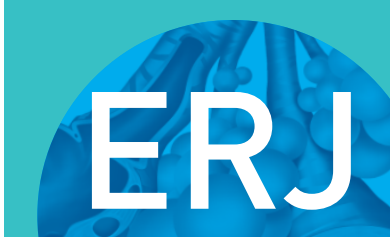

open research

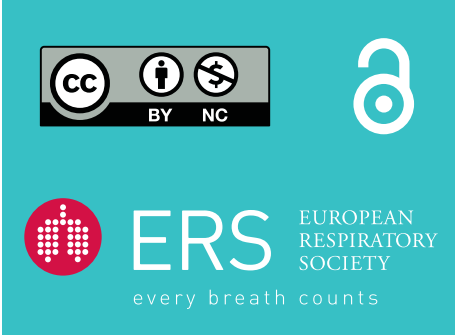

\section{Conservative management of COVID-19 associated hypoxaemia}

\section{To the Editor:}

With appropriate esteem, we have read the article by VOSHAAR et al. [1] on the management of hypoxaemia related to COVID-19 pneumonia. The authors have concluded that treatment escalation in the sequence 1) supplemental oxygen; 2) nasal high-flow, continuous positive airway pressure (CPAP) or noninvasive ventilation (NIV); and 3) invasive mechanical ventilation resulted in low overall mortality if guided by the calculation of oxygen content in addition to the clinical presentation. We fully agree with the authors and others $[2,3]$ that hypoxaemia per se should not serve as the exclusive indication parameter for intubation. However, we disagree that this statement is, in any manner, supported by the data presented by VoshaAr et al. [1].

Noninvasive strategies were escalated to intubation if "clinical work of breathing required the next level of support or the oxygen content was determined to be below $9 \mathrm{~mL} \mathrm{O}_{2} \cdot \mathrm{dL}^{-1}$ ". Unfortunately, "clinical work of breathing" was not defined at all. Neither was oesophageal pressure measured, nor was arterial carbon dioxide tension or information on phasically increased contractions of respiratory muscles, or any other parameter indicative of the work of breathing [4] provided. In addition, the mean oxygen content in patients having experienced intubation was $11.6 \pm 2.6 \mathrm{~mL} \cdot \mathrm{dL}^{-1}$. Thus, no patient seems to have met the preset cut-off. Sadly, it remains fully unclear based on what criteria patients had been eventually intubated. The final conclusion that "a respiratory support escalation scheme based on clinical appearance and oxygen... has a favourable outcome" is certainly not supported by the current findings.

Per the designated protocol, patients with an oxygen saturation $<93 \%$ were included but true mean saturation was reportedly $92 \pm 6 \%$, indicating that assuming normal distribution, nearly half of the patients had an oxygen saturation $\geqslant 93 \%$. Table 2 and the figures list several parameters but it remains unclear when and under what circumstances (under treatment?) values were obtained. Thus, it remains unclear what type of protocol has effectively existed. It also remains speculative when and under what conditions haemoglobin values and the corresponding arterial oxygen tension $\left(P_{\mathrm{aO}_{2}}\right)$ values were determined. Presenting nadir haemoglobin and nadir $P_{\mathrm{aO}_{2}}$ values suggests that both values were not measured correspondingly but rather at staggered intervals. Based on this, the actual oxygen content is suggested to be artificially low.

It is also implied that current recommendations still suggest intubation in severe COVID-19 pneumonia primarily based on markers of oxygenation. Here, the authors refer to historical German guidelines but, unfortunately, ignore more recent updates that have already emphasised the importance of considering several clinical criteria in addition to markers of oxygenation refractory to noninvasive treatment modalities, in order to individually target the decision to intubate the patient rather than purely to guide the decision in view of oxygenation [4-6].

In addition, the authors mention most recent trials on the usefulness of noninvasive treatment strategies $[7,8]$, but they expound on basic physiological contexts rather than discuss their findings in more detail in view of the previous findings. Here, the long-winded explanations on physiology give the impression of an emphasised importance of considering physiological interrelations in clinical practice, but in fact, the authors present themselves physiologically imprecisely. As an example, they indicate to have monitored oxygen saturation by pulse oximetry $\left(S_{\mathrm{pO}_{2}}\right)$ but, contrarily, they had provided data on arterial oxygen

@ERSpublications

This correspondence argues that the conclusion given in the article "Conservative management of COVID-19-associated hypoxaemia" is not supported by the data https://bit.ly/3qAn7la

Cite this article as: Windisch W, Kluge S, Bachmann $\mathrm{M}$, et al. Conservative management of COVID-19 associated hypoxaemia. ERJ Open Res 2021; 7: 00113-2021 [https://doi.org/10.1183/ 23120541.00113-2021].

Copyright $\odot$ The authors 2021. This version is distributed under the terms of the Creative Commons Attribution NonCommercial Licence 4.0. For commercial reproduction rights and permissions contact permissions@ersnet.org 
saturation $\left(S_{\mathrm{aO}_{2}}\right)$ exclusively in their accepted paper, even though this has been verified after proof correction. This also ignores differences between $S_{\mathrm{pO}_{2}}$ and $S_{\mathrm{aO}}$ as particularly established for hypoxaemic COVID-19 patients [9]. Furthermore, it is not clear how the inspiratory fraction of oxygen was determined in patients spontaneously breathing in a retrospective approach.

We certainly do not speak against noninvasive treatment modalities, i.e. high-flow oxygen, CPAP or NIV, for managing severe COVID-19 disease. Furthermore, we strongly believe that noninvasive treatment modalities and invasive ventilation are complementary rather than competing treatment options in the hands of wise and experienced physicians who do not regard oxygenation as the primary target of the important decision of intubation [4]. Given the established complication rates, intubation should certainly be avoided by all available means [2-8].

This has been successfully achieved by CPAP according to two recently published studies $[7,8]$. The current study namely targets this important issue but remains inconclusive for the given concerns. Measurement dates have been provided not at all. The manuscript includes several substantive errors. In response to our remarks, the authors have, fortunately, corrected some of these. Finally, it remains unclear how many patients were treated on intermediate care and intensive care units (ICUs). Two thirds of the patients received oxygen only, forming a cohort regularly treated on the normal floor, while information on scores typically used to characterise critically ill patients are not given. Despite this, the authors compare their data with real ICU patients and, in the end, are prone to "compare apples and oranges".

Wolfram Windisch $^{1}$, Stefan Kluge ${ }^{2}$, Martin Bachmann ${ }^{3}$, Carl-Peter Criée ${ }^{4}$, Steffen Weber-Carstens $\oplus^{5}$, Michael Westhoff ${ }^{6}$, Christian Karagiannidis $^{1}$ and Tobias Welte $\odot^{7}$

${ }^{1}$ Cologne Merheim Hospital, Dept of Pneumology, Kliniken der Stadt Köln gGmbH, Witten/Herdecke University, Cologne, Germany. ${ }^{2}$ Dept of Intensive Care, University Medical Center Hamburg-Eppendorf, Hamburg, Germany. ${ }^{3}$ Dept of Intensive Care and Ventilatory Medicine, Asklepios Klinikum HamburgHarburg, Hamburg, Germany. ${ }^{4}$ Dept of Pneumology and Mechancial Ventilation, Ev. Krankenhaus Göttingen-Weende e.V., Beatmungsmedizin und Schlaflabor, Bovenden-Lenglern, Germany. ${ }^{5}$ Dept of Anesthesiology and Operative Intensive Care Medicine, Charité (Universitätsmedizin Berlin), corporate member of Freie Universität Berlin, Humboldt Universität zu Berlin and Berlin Institute of Health, Berlin, Germany. ${ }^{6}$ Dept of Respiratory Medicine, Hemer Lung Hospital, Witten/Herdecke University, Hemer, Germany. ${ }^{7}$ Dept of Respiratory Medicine and German Centre of Lung Research (DZL), Hannover Medical School, Hannover, Germany.

Correspondence: Wolfram Windisch, Dept of Pneumology, Lung Clinic, Cologne Merheim Hospital, Witten/Herdecke University, Ostmerheimerstraße 200, 51103 Cologne, Germany.

E-mail: windischw@kliniken-koeln.de

Received: 15 Feb 2021 | Accepted: 4 March 2021

Conflict of interest: W. Windisch's hospital has received research grants from Respironics USA, Löwenstein Medical Germany and VitalAire Germany; and he has received personal speaking fees from companies dealing with mechanical ventilation. S. Kluge has received research grants from Ambu, Daiichi Sankyo, ETView Ltd, Fisher and Paychel, and Xenios; consulting fees from Bayer, Fresenius, Gilead, MSD and Pfizer; and lecture fees from Astra, CR Bard, Baxter, Biotest, Cytosorbents, Daiichi Sankyo, Fresenius, Gilead, MSD, Pfizer, Philips and Zoll. M. Bachmann has received consulting fees from companies dealing with mechanical ventilation and payment for a presentation from Philips Respironics; and is the president of the German Interdisciplinary Society for Home Mechanical Ventilation (DIGAB). C-P. Criée has nothing to disclose. S. Weber-Carstens' hospital has received a research grant from Dräger Medical; and he has received personal speaking fees from companies dealing with mechanical ventilation and extracorporeal membrane oxygenation. M. Westhoff s hospital has received a grant from Löwenstein Medical Germany; and he has received consulting fees from companies dealing with mechanical ventilation, and payments or honoraria for lectures, presentations, speakers bureaus, manuscript writing or educational events from Löwenstein Medical Germany. C. Karagiannidis has received a research grant from the German Ministry of Research and Education; consulting fees from companies dealing with mechanical ventilation; and speaking fees from Maquet Germany, Xenios Germany and Bayer Germany; and is the organizer of the Germany Registry of ICUs. T. Welte has nothing to disclose.

\section{References}

1 Voshaar T, Stais P, Köhler D, et al. Conservative management of COVID-19 associated hypoxaemia. ERJ Open Res 2021; 7: 00026-2021.

2 Tobin MJ, Jubran A, Laghi F. Hypoxaemia does not necessitate tracheal intubation in COVID-19 patients. $\mathrm{Br} \mathrm{J}$ Anaesth 2021; 126: e75-e76.

3 Tobin MJ, Jubran A, Laghi F. Noninvasive strategies in COVID-19: epistemology, randomised trials, guidelines, physiology. Eur Respir J 2020; 57: 2004247.

4 Windisch W, Weber-Carstens S, Kluge S, et al. Invasive and non-invasive ventilation in patients with COVID-19. Dtsch Arztebl Int 2020; 117: 528-533. 
5 Pfeifer M, Ewig S, Voshaar T, et al. Position paper for the state-of-the-art application of respiratory support in patients with COVID-19. Respiration 2020; 99: 521-542.

6 Kluge S, Janssens U, Welte T, et al. S2k guideline - recommendations for inpatient therapy of patients with COVID-19. Pneumologie 2021; 75: 88-112.

7 Brusasco C, Corradi F, Di Domenico A, et al. Continuous positive airway pressure in Covid-19 patients with moderate-to-severe respiratory failure. Eur Respir J 2020; 57: 2002524.

8 Oranger M, Gonzalez-Bermejo J, Dacosta-Noble P, et al. Continuous positive airway pressure to avoid intubation in SARS-CoV-2 pneumonia: a two-period retrospective case-control study. Eur Respir J 2020; 56: 2001692.

9 Sjoding MW, Dickson RP, Iwashyna TJ, et al. Racial bias in pulse oximetry measurement. N Engl J Med 2020; 383: 2477-2478. 